\title{
Balkanologie
}

Balkanologie Revue d'études pluridisciplinaires

Vol. V, no 1-2 | 2001

Volume V Numéro 1-2

\section{Sarajevo, capitale incertaine}

\section{Amaël Cattaruzza}

\section{OpenEdition}

Journals

Édition électronique

URL : http://journals.openedition.org/balkanologie/685

DOI : 10.4000/balkanologie.685

ISSN : 1965-0582

\section{Éditeur}

Association française d'études sur les Balkans (Afebalk)

Édition imprimée

Date de publication : 31 décembre 2001

ISSN : 1279-7952

\section{Référence électronique}

Amaël Cattaruzza, «Sarajevo, capitale incertaine », Balkanologie [En ligne], Vol. V, n 1-2 | 2001, mis en ligne le 31 mai 2008, consulté le 17 décembre 2020. URL : http://journals.openedition.org/ balkanologie/685; DOI : https://doi.org/10.4000/balkanologie.685

Ce document a été généré automatiquement le 17 décembre 2020.

(c) Tous droits réservés 


\title{
Sarajevo, capitale incertaine
}

\author{
Amaël Cattaruzza
}

1 Sarajevo a occupé au cours du XXe siècle une place particulière dans la mémoire collective occidentale. Faut-il croire que le traumatisme causé par l'assassinat de François Ferdinand, Archiduc d'Autriche, le 28 juin 1914, ne s'est jamais vraiment effacé chez les peuples d'Europe de l'Ouest? Ce souvenir passionnel et tragique n'aurait-il pas en partie accentué l'engouement des journalistes, intellectuels et hommes politiques qui, pendant les quatre années du conflit bosniaque, ont fait de cette ville le symbole d'une résistance contre les nationalismes en guerre, et celui d'un espoir de coexistence intercommunautaire face à la barbarie de la "purification ethnique »? Mais aujourd'hui, que reste-t-il de tout cela? Dans cet article, nous nous proposons de dresser un bilan de la situation complexe de cette ville en l'an 2000, cinq ans après le retour de la paix.

2 Sarajevo est d'abord une ville qui a connu un siège d'un peu moins de quatre ans, entre avril 1992 et décembre 1995, période pendant laquelle elle n'a pas cessé d'être pilonnée, et où ses habitants étaient constamment menacés. La guerre a profondément affecté la structure de sa population, une population à près de $85 \%$ bochniaque aujourd'hui ( $49 \%$ en 1991), du fait des déplacements forcés qui ont été le but avoué du conflit. Cette nouvelle répartition suffit à ébranler le mythe qui voudrait que Sarajevo soit restée une cité pluriculturelle et pluriethnique, une "Jérusalem balkanique". En outre, à la dissociation ethnique viennent s'ajouter d'autres types de fractures. Avec l'arrivée massive de réfugiés dans la ville pendant et après le conflit, des tensions sont apparues entre les "désabusés", anciens Sarajéviens ayant vu leur ville détruite jour après jour, et les "déracinés", nouveaux Sarajéviens issus la plupart du temps d'un milieu rural ou de villes plus petites, et qui se heurtent à des coutumes urbaines, des civilités qu'ils ne connaissent pas. L'incompréhension entre ces deux groupes est accrue par un contexte social difficile dans lequel le marché noir fleurit, seul moyen de subsistance pour de nombreuses personnes sans travail et sans ressource.

3 Dans une perspective plus générale, cette ville est en transition entre système communiste et démocratie libérale, à l'image des pays de l'Est dans leur ensemble. Ce problème doit toujours être placé en toile de fond. En effet, derrière la réparation des 
vestiges de la guerre, c'est tout un système de valeurs qui est en train d'évoluer, de se transformer. L'ampleur de ce changement suffit à expliquer certains blocages, tant au niveau économique et administratif (industries et administrations dominées par les partis politiques) qu'au niveau individuel (nostalgie de la période titiste).

Enfin, Sarajevo est, depuis 1992, une capitale de rang international à la tête d'un nouvel Etat indépendant, la Bosnie-Herzégovine. Cette position dominante de Sarajevo à l'intérieur du pays est plutôt récente, et reste sujette à contestation. Aussi, la ville doit trouver sa place tant au niveau national qu'international, à l'heure où elle traverse de grandes difficultés politiques et économiques. Sur la scène mondiale, elle reste sous tutelle des différentes organisations internationales en présence. Sur le plan national, elle est soumise à la fragilité de l'Etat bosniaque. En effet, le pays est actuellement divisé en trois zones distinctes qui ne coopèrent que très peu ensemble : deux entités créées par les accords de Dayton (décembre 1995) et séparées par une ligne interentités, la Republika Srpska (République serbe) et la Fédération croato-bochniaque, et au sein de cette dernière, deux zones croate et bochniaque. Or, la ville de Sarajevo a été âprement disputée pendant le conflit, et se situe désormais en grande partie en zone bochniaque, mais jouxte la Republika Srpska. Dans ce contexte, nous verrons que la place de Sarajevo est donc bien précaire et pleine d'incertitudes.

\section{Sarajevo, une histoire démographique mouvementée}

5 L'installation humaine dans la région de Sarajevo est ancienne. Celle-ci présente en effet l'avantage d'être située dans la vaste vallée de Sarajevo-Zenica, à proximité du point de confluence de la Miljačka et de la Bosna, c'est-à-dire dans un site qui était prédisposé à être, dans cet espace montagneux, un lieu de passage et de communication, un carrefour entre l'Europe de l'Est et la côte Adriatique, entre Orient et Occident. Au Moyen Âge, alors que la Bosnie devenait progressivement un royaume indépendant, on voit quelques cités fortifiées (Hodidjed, Kotorac, Vrhbosna, Trgovište) s'établir aux alentours du site actuel de Sarajevo.

Depuis sa fondation en 1462 par Isa Beg Isaković à l'époque gouverneur de BosnieHerzégovine, Sarajevo a toujours été soumise à des systèmes politiques larges (Empires, Royaume ou Fédération) qui ont pu favoriser, par leur fonctionnement ou dans leur succession, un brassage communautaire important. A travers l'histoire de Sarajevo, nous pouvons distinguer différentes étapes de son développement urbain et démographique : la période de domination ottomane, celle de l'administration austrohongroise, celle du Royaume des Serbes, Croates et Slovènes, et enfin celle de la Yougoslavie de Tito ${ }^{1}$.

7 La ville ne connaît un réel essor qu'au début du XVIe siècle, lorsque Gazi Husrev Beg devient, à son tour, gouverneur de Bosnie. Il décide de faire de ce centre administratif un šeher ("grande ville"), et y fait construire de nombreux monuments et édifices publiques. On lui doit la plus belle mosquée de Sarajevo, qui porte son nom (Begova džamija, 1530), une bibliothèque (1537), une medressa (école coranique, 1538), un bezistan (marché couvert, 1542), etc. Le XVIème siècle est l'âge d'or de la ville. Le système ottoman est suffisamment souple pour favoriser les échanges commerciaux et la pluriculturalité dans la cité. Le principe du millet permet à chaque communauté de rester sous l'autorité de son propre chef religieux. A Sarajevo, on dénombre à la fin du siècle quatre-vingt onze mahale (quartiers) musulmans, deux chrétiens, ainsi qu'une 
colonie juive. La domination ottomane se traduit dans les villes par de nombreuses conversions à l'islam. Le nombre d'habitants musulmans à Sarajevo, ou du moins déclarés comme tels, augmente rapidement. La présence d'une communauté juive joue un rôle important dans la croissance de l'activité commerciale, et notamment du commerce extérieur, en raison de ses liens avec d'autres colonies et des personnalités juives de l'Empire et des pays occidentaux. Une synagogue est construite vers 1580 . Sarajevo reste sous autorité ottomane pendant plus de quatre cents ans, de 1462 à 1878.

Le 13 août 1878, le Congrès de Berlin confie l'administration de la Bosnie-Herzégovine à l'Autriche-Hongrie. La période de domination austro-hongroise, qui ne dure que quarante ans, inaugure un second stade de développement de la ville. Des bâtiments d'un style nouveau, plus académique, commencent à côtoyer les anciens quartiers ottomans. Le nombre des catholiques augmente, justifiant l'édification d'une cathédrale. Cet accroissement de la population catholique est en partie dûe a une politique délibérée de l'administration austro-hongroise, comme en témoignent la création en 1881 de l'archevêché de Sarajevo, des évêchés de Banja Luka et de Mostar, et la nomination au siège épiscopal de Josip Stadler, un catholique ultramontain. La nouvelle structure démographique mise en place à Sarajevo au cours des quarante ans de tutelle austro-hongroise reste encore perceptible après la Première Guerre mondiale, à l'époque du Royaume des Serbes, Croates et Slovènes, même si la part des Serbes (orthodoxes) augmente alors au détriment de la population croate (catholique).

9 La troisième grande phase d'extension et de développement de la ville remonte au temps de la Yougoslavie communiste. L'agglomération continue alors de s'étendre vers l'ouest le long de la vallée de la Miljačka. Sa population augmente de façon constante jusqu'au déclenchement de la guerre, confortant son caractère multiculturel mais bouleversant une fois encore ses structures démographiques. En 1991, au cours du dernier recensement yougoslave, le nombre de Sarajéviens s'élève à 527049 , dont 49,2\% de Musulmans (Bochniaques), 29,8\% de Serbes, 6,6\% de Croates, 10,7\% de "Yougoslaves", et 3,6 \% d'habitants classés dans la catégorie "Autres". Le nombre de Musulmans s'est donc accru jusqu'à représenter près de la moitié des habitants, tandis que l'accroissement du nombre de personnes s'affirmant "Yougoslaves" rend plus délicate une quelconque analyse de l'évolution relative des populations serbe et croate. Toujours est-il que la ville est alors considérée comme une illustration de la réussite du modèle d'intégration yougoslave et, de fait, les mariages mixtes y sont plus courants que dans les campagnes, sans toutefois se généraliser.

L'attention que nous portons volontairement aux différentes appartenances communautaires ne doit pas cacher d'autres types de disparités, comme celles qui apparaissent au niveau social. Elles expliquent l'apparition dans les années 1970 d'une banlieue ouvrière, formée de grandes tours d'habitation, alors que Sarajevo commence à sentir les effets de la crise économique yougoslave. Les Jeux Olympiques d'hiver en 1984 lui permettent cependant d'atténuer les retombées de cette crise. De grands travaux de rénovation et de restructuration urbaine y sont alors accomplis. La croissance de la ville se maintient péniblement. Elle est brutalement stoppée en avril 1992, avec le début du siège. 


\section{Les nouvelles structures démographiques, sociales et identitaires}

11 Les logiques communautaires, religieuses et nationalistes de la guerre ont profondément affecté Sarajevo, tant au niveau démographique qu'au niveau identitaire et social. Les estimations du Haut commissariat aux réfugiés, datées de juillet 1999 pour Sarajevo et de mars 1996 pour Srpsko Sarajevo ("Sarajevo serbe"), indique que la ville serait peuplée de 361934 habitants côté Fédération (dont 59685 personnes déplacées), et de 29689 personnes côté serbe, soit, dans le meilleur des cas, d'un total de 391623 habitants. L'inversion de tendance démographique est évidente sur une courbe :

Evolution démographique de Sarajevo entre 1948 et 1999

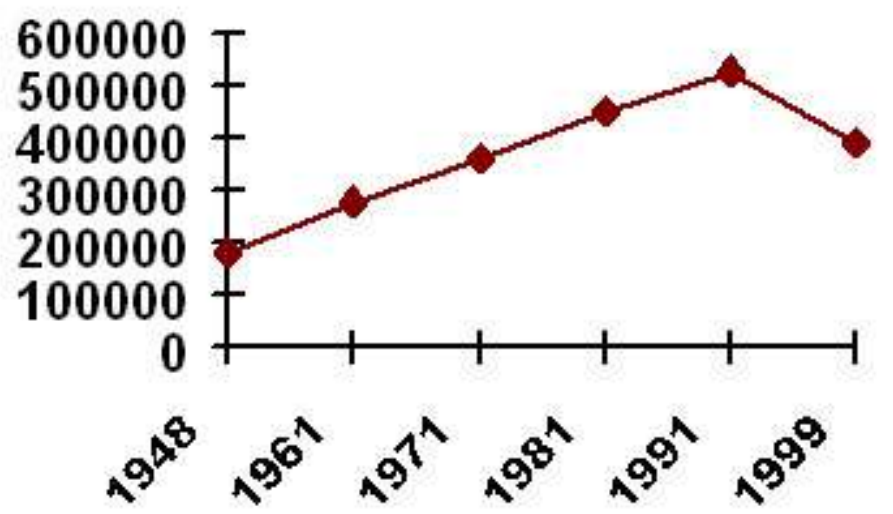

En 1999, la ville est presque revenue à son niveau de 1971. Les raisons de cette perte sont doubles : d'une part, les morts, tués par les obus ou les snipers (le bilan humain de la guerre est estimé à 10000 morts environ pour Sarajevo), d'autre part, les personnes déplacées, familles serbes, croates, mixtes, ou même bochniaques qui ont dû fuir la ville. La partie de Sarajevo située en Fédération serait peuplée à $84 \%$ environ de Bochniaques, alors qu'ils n'y représentaient que 49,2 \% en 1991. Les minorités ethniques, serbe ou croate, sont aujourd'hui regroupées dans le centre-ville. Dans la partie serbe de Sarajevo, il n'existe pas de données exactes concernant les différentes communautés ; cependant, nous pouvons affirmer que la quasi-totalité de la population est serbe.

Pour contrecarrer cette homogénéisation ethnique de la ville, les organisations internationales mettent l'accent sur le retour des réfugiés. L'Office du Haut représentant de l'ONU (Office of the High Representative-OHR) a ainsi appelé les institutions internationales à signer le 3 février 1998 une Déclaration de Sarajevo. Celle-ci présente les priorités de la communauté internationale pour l'agglomération et son canton, à savoir la multiethnicité, la non-discrimination, le respect de la propriété privée des personnes déplacées et un nombre d'au moins 20000 retours minoritaires en $1998^{2}$. Cette année-là, le Haut commissariat aux réfugiés n'a en fait enregistré que 4934 retours minoritaires dans le canton de Sarajevo, auxquels s'ajoutent les retours spontanés qui peuvent atteindre quelques milliers. Quoi qu'il en soit, l'objectif de 
départ est très loin d'avoir été atteint, car les entraves au retour sont nombreuses. Tout d'abord, le processus démographique amorcé par les accords de Dayton semble ne pas aller dans le sens d'une réintégration humaine mais, au contraire, dans celui d'une dissociation ethnique et territoriale de plus en plus marquée. Louis Sell évoque ainsi le départ massif des Serbes de Sarajevo après la fin du siège et le départ de l'armée assiégeante ${ }^{3}$. Sur les quelques 157000 Sarajéviens serbes d'avant-guerre, il estime qu'il en restait 10000 seulement à la fin du retrait des forces serbes, la plupart étant partis s'installer en Republika Srpska. Quant à ceux qui désireraient maintenant rentrer dans leur ancien foyer, la démarche apparait longue, fastidieuse et risquée du fait de l'obstruction délibérée des administrations (l'influence du SDA -parti nationaliste musulman- y est prégnante), de la discrimination à l'embauche, dans l'éducation, la santé, etc., et des problèmes identitaires qu'un tel retour implique. Or, plus le temps passe, plus les retours minoritaires semblent hypothétiques.

Pourtant, la situation sociale des Sarajéviens est globalement plus enviable que celle du reste du pays. Le taux de chômage au 1er janvier 2000 serait à Sarajevo de 14,4 \%, avec 52110 chômeurs, alors qu'il est de plus de 50 \% en Bosnie-Herzégovine. Néanmoins, au nombre officiel de chômeurs, il faut ajouter les travailleurs "en attente" (na čekanju) qui sont employés par des entreprises n'ayant pas réouvert depuis la guerre. Le taux de chômage atteint alors 18 \% environ, c'est-à-dire près de un actif sur cinq. La proportion de chômeurs est moindre lorsque l'on s'approche du centre ville. Cette différence est peut-être dûe à la concentration dans le centre du secteur tertiaire, qui représente probablement le principal fournisseur d'emploi de la capitale. Mais la condition des travailleurs n'est pas bien meilleure que celle des chômeurs. D'après les sources officielles, le revenu moyen mensuel d'un Sarajévien serait en janvier 2000 de 466,74 KM (Mark convertible), soit l'équivalent de $1570 \mathrm{FF}$ ou 236 Euros $^{4}$. La situation précaire d'un grand nombre d'habitants explique le développement d'un important marché noir, qui est en fait presque légal, puisqu'il se fait à la vue de tous, dans la rue, sur les marchés ou au porte-à-porte. Les fractures qui divisent la population sarajévienne pourraient donc être bien plus de nature sociale qu'ethnique.

En effet, les tensions nationalistes se trouvent atténuées à Sarajevo par rapport à l'ensemble du pays, peut-être du fait d'une identité sarajévienne particulière. Celle-ci passe d'abord par l'apprentissage de toute une mythologie qui tourne autour des différents sites de la ville, et le respect de certains "rites", comme celui de se retrouver le $1^{\text {er }}$ mai à Ilidža, aux sources de la Bosna, pour célébrer la fête du travail. Elle se fonde enfin sur l'histoire de la ville, connue et partagée par tous les Sarajéviens. Or, cette identité est aujourd'hui remise en cause par l'afflux de nouveaux arrivants. Si l'écart entre l'ancienne et les nouvelles populations est important à Sarajevo, il devient plus profond encore lorsque les nouveaux arrivants sont d'origine rurale. Deux cultures se côtoient et s'affrontent alors : culture urbaine et culture rurale.

Les noms ne manquent pas chez les anciens Sarajéviens pour qualifier ces ruraux : les primitivci ou encore les papci (sing. : papak), la traduction n'étant pas nécessaire pour deviner l'aspect péjoratif de ces termes. Aujourd'hui, il existe à Sarajevo un conflit latent entre les "déracinés", ces personnes arrachées à leur campagne, qui se retrouvent perdues en ville, et les "désabusés", ces Sarajéviens qui ont d'abord vu leur ville détruite et qui voient aujourd'hui de nouvelles populations mettre en péril, par des comportements nuisibles à la ville, l'intégrité de leur cité. Ce phénomène d'exode rural n'est pas nouveau, mais il a été considérablement accru par la guerre. Et cette 
arrivée soudaine et massive de ruraux au sein de la capitale pose de nouveaux problèmes : les papci vont-il s'intégrer à la ville, ou le tissu social de Sarajevo va-t-il se désintégrer faute de résoudre cette tension? Va-t-on assister à l'émergence d'un papak citadin, ou à une communautarisation accrue de la population de Sarajevo ?

\section{Une capitale contestée au niveau national}

17 Sarajevo est, depuis l'indépendance de la Bosnie-Herzégovine, la capitale d'un nouvel Etat. Elle est également, depuis les accords de Washington (mars 1994), la capitale de la Fédération croato-bochniaque. Mais ce double statut est loin d'être acquis pour la ville, et les prochains défis que Sarajevo va devoir relever pour assumer pleinement sa place de capitale vont être de s'affirmer tant au niveau national qu'international.

Il lui faudra d'abord être reconnue par l'ensemble du pays, ce qui repose le douloureux problème de la division de la Bosnie-Herzégovine. Cette reconnaissance paraît d'autant plus difficile à obtenir que la ville a représenté un enjeu important au cours du conflit. Trois plans de paix ont échoué en partie sur la question du statut de Sarajevo, car ils proposaient de faire passer la ville sous administration internationale ${ }^{5}$. Finalement, les accords de Dayton (décembre 1995) ont mis fin à l'encerclement de la ville par les troupes serbes et accordé la plus grande partie de son territoire à la Fédération croatobochniaque, réduisant la zone serbe à quelques secteurs périphériques à l'est et au sud de la ville. Cette solution était à l'époque bien loin de satisfaire les dirigeants bosnoserbes qui auraient préféré une division plus "équitable", et leur ressentiment est encore perceptible aujourd'hui dans le mépris qu'ils ont envers leur capitale. Le statut de capitale octroyé à Sarajevo reste donc fragile, par la définition même de ses pouvoirs dans les accords de paix, et par sa contestation dans une partie du pays. En outre, la ville doit aussi s'émanciper de la tutelle internationale, ce qui semble bien loin de se réaliser, tant au niveau économique que politique et militaire.

Au niveau de la Bosnie-Herzégovine, les pouvoirs attribués par les accords de Dayton au gouvernement central installé à Sarajevo sont limités aux affaires étrangères, et aux questions intérieures qui nécessitent une harmonisation globale comme la monnaie, les communications et les transports. La capitale n'est donc pas le lieu de toutes les décisions dans le pays, et les accords de Dayton ont rejeté toute solution allant dans le sens d'une centralisation plus poussée. Au niveau de la Fédération croato-bochniaque, les pouvoirs de Sarajevo sont également restreints: chaque canton vote ses propres lois, possède ses propres administrations et sa propre police, et élabore ses propres programmes scolaires et universitaires. La mise en place de la Fédération s'accompagne donc d'un formidable processus de décentralisation des pouvoirs. La parité entre représentants bochniaque et croate est scrupuleusement respectée au sein des institutions fédérales, ce qui révèle le principe idéologique sous-jacent à la création de la Fédération : la division. De fait, les cantons ne coopèrent que très peu entre eux.

Un autre problème qui se pose est la lourdeur que cet enchevêtrement institutionnel et administratif implique. En effet, chaque canton a son ou ses Président(s), son Assemblée et son gouvernement, tout comme la Fédération croato-bochniaque et la Bosnie-Herzégovine elle-même. A ces trois échelons de pouvoir s'ajoute celui des municipalités. Sarajevo, située au sommet de cette pyramide, est devenue une ville où les administrations ont pris une importance démesurée, puisque les quatre échelons s'y trouvent concentrés sur un territoire restreint. En outre, la mise en place du canton de 
Sarajevo en mars 1996 a eu pour conséquence une perte de compétences et de revenus importante pour la municipalité.

21 Sarajevo est encore loin de pouvoir se prévaloir d'une reconnaissance unanime de la part de la population bosniaque. La contestation que subit la ville se fait sur des bases soit régionales, soit ethniques. Sur le plan régional, la concurrence entre les différentes villes bosniaques est ancienne. Notons que la capitale politique de la Bosnie au temps de la domination ottomane n'était pas Sarajevo, mais Travnik. Au cours du XXe siècle, Sarajevo s'est sans doute développée plus vite que n'importe quelle autre ville de Bosnie-Herzégovine, du fait des politiques délibérées de l'Empire austro-hongrois, du Royaume des Serbes, Croates et Slovènes, et à leur suite de la Yougoslavie communiste. Mais cette centralité de Sarajevo n'a cessé de susciter la jalousie des autres centres urbains tels que Banja Luka, Mostar, Tuzla ou Zenica. Celle-ci s'est notamment manifestée en 1984, lorsque que d'importants investissements ont été réalisés à Sarajevo pour les Jeux Olympiques d'hiver. Sur le plan ethnique, les zones croates sont plus tournées vers la Croatie, avec laquelle elles partagent des frontières communes, que vers Sarajevo. Quant à la Republika Srpska, elle possède ses propres centres régionaux auxquels elle accorde un statut de "capitales" : d'un côté, Banja Luka, qui est devenue le centre administratif de la Republika Srpska, et de l'autre, Srpsko Sarajevo, la partie serbe de Sarajevo séparée de la capitale par la Ligne-frontière inter-entités (Inter-Entity Boundary Line -IEBL).

Or, l'IEBL qui traverse actuellement Sarajevo pose un problème tout aussi grave que celui de sa reconnaissance en tant que capitale : cette ville n'est-elle pas en train de devenir une ville-frontière? Parler de ville-frontière en ce qui concerne Sarajevo reste contestable, car cela demande au préalable de considérer l'IEBL comme une frontière, alors que celle-ci n'est pas véritablement reconnue comme telle par les autorités nationales et internationales. Elle est définie par les accords de Dayton comme une simple « ligne de démarcation entre les entités », et aucun autre statut ne lui est donné. Pourtant, cette ligne agit dans les faits comme une frontière, et qui plus est une frontière fermée. Dans ces conditions, la zone d'influence de la ville s'arrête de façon très claire à l'IEBL. Cette position pourrait être dangereuse pour l'avenir économique à long terme de la capitale : Sarajevo ne risque-t-elle pas d'être marginalisée au sein de la Fédération croato-bochniaque, alors même qu'elle se situe au coeur de la BosnieHerzégovine? Cette situation de ville-frontière pourrait ausi avoir des conséquences politiques graves à long terme, la ville se repliant sur elle-même et s'homogénéisant toujours plus sur le plan ethnique pour se protéger du danger que représente la proximité de la Republika Srpska. Elle deviendrait alors un "rempart bochniaque" opposé aux zones serbe et croate. De fait, les industries situées dans la capitale restent au mains du SDA, le parti nationaliste musulman, et les mosquées se multiplient dans le but de stabiliser la nouvelle composition ethnique de la ville. De l'autre côté, Srpsko Sarajevo affirme par tous les moyens son identité serbe. Sarajevo et Srpsko Sarajevo multiplient donc les provocations réciproques comme l'érection de monuments à leur héros de guerre respectifs, les discriminations de tous ordres et le mépris mutuel de leurs habitants.

Srpsko Sarajevo est une création issue de la guerre, sans aucun fondement historique. Elle est consignée dans la zone attribuée à la Republika Srpska par les accords de Dayton, qui s'étend sur quelques quartiers et secteurs périphériques à l'Est et au Sud de Sarajevo. Le projet serbe d'un Srpsko Sarajevo pouvant rivaliser avec la capitale s'inscrit 
dans la perspective du rattachement des quelques quartiers serbes (Dobrinja, Lukavica) à la petite ville de Pale, vingt kilomètres plus à l'Est. Aussi, les autorités serbes se sont efforcées avec le peu de moyens dont elles disposaient de reconstruire les bâtiments des quartiers serbes de Sarajevo et de favoriser l'essor de Pale, afin de permettre aux réfugiés serbes de s'installer définitivement, et de pérenniser ainsi l'homogénéisation ethnique de ce territoire. Mais ces efforts de développement cachent une réalité assez déplorable : le niveau économique de Srpsko Sarajevo est nettement inférieur à celui de Sarajevo, et les reconstructions sont beaucoup moins avancées du côté serbe. Les politiques de reconstruction des organisations internationales ont nettement favorisé la Fédération : officieusement, $82 \%$ des aides seraient allées à la Fédération, contre $18 \%$ seulement à la Republika Srpska.
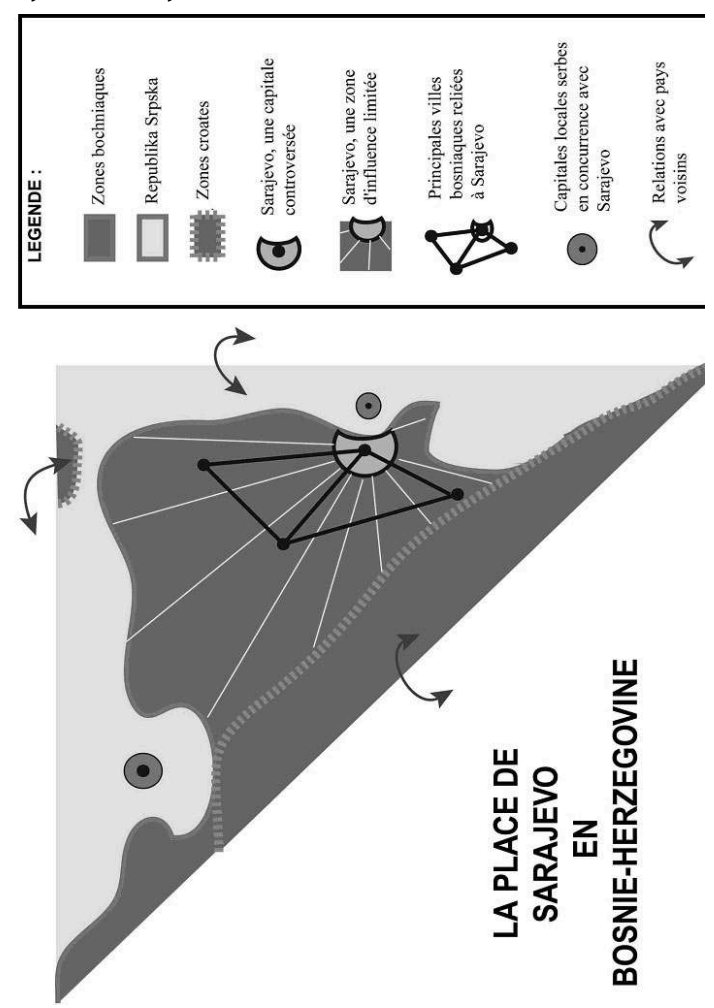

\section{Une forte présence internationale}

$\mathrm{Au}$ niveau international, Sarajevo est une ville sous tutelle. A l'aéroport de Sarajevo, placé sous la surveillance de la SFOR (Stabilization Force), avions civils et avions de transport militaires se partagent les pistes. En ville, des soldats sillonnent en permanence les rues, et des véhicules militaires de toutes sortes se mêlent aux voitures ordinaires. Cette présence de la SFOR en Bosnie-Herzégovine a pour principal objectif de prévenir la reprise éventuelle d'un conflit, et de soutenir les efforts de la communauté internationale. Un retrait progressif a déjà été amorcé, mais on peut penser qu'il ne s'achèvera pas avant longtemps.

La présence internationale est également visible au niveau politique, à travers les interventions de l'Office du Haut représentant de l'ONU (OHR). Pour la ville de Sarajevo, cette tutelle politique se traduit par exemple par le fait que, le 29 novembre 1999, le Haut représentant a destitué vingt-deux élus ou responsables politiques locaux, dont le maire de la partie serbe d'Ilidža (située dans Srpsko Sarajevo) et le ministre de la Justice 
du canton de Sarajevo. Plus largement, le but avoué de cette tutelle internationale est la conversion de la Bosnie-Herzégovine à la démocratie libérale. Ce n'est là qu'une tentative de "rectification" politique dans un pays qui connaît le règne de la "partitocratie". Et, de fait, certains rapports internationaux soulignent la faible influence de la communauté internationale comparée à la domination, à toutes les échelles de décision, des partis nationalistes.

$\mathrm{Au}$ niveau financier, la ville doit également beaucoup à la participation internationale. Les fonds accordés à la Bosnie-Herzégovine sous forme de dons ou de crédits seraient, depuis la fin de la guerre, de l'ordre de 5,2 milliards de dollars. Autant dire que peu de choses auraient pu être réalisées sans cette aide, qui a toutefois un prix, celui de rendre le pays et sa capitale débiteurs pour de nombreuses années. Cette dépendance s'exprime également sur le plan technique, puisque les organisations étrangères s'occupent aussi de l'encadrement et du suivi des projets qu'elles financent. Ce sont elles qui fournissent l'essentiel des ingénieurs et des techniciens qui gèrent les reconstructions.

Cette implication étrangère tous azimuts se traduit, dans la capitale, par une présence massive de gens d'origines diverses, mais principalement occidentales, qui vivent le plus souvent en autarcie, au sein d'une micro-société se renouvelant tout les six mois. Leurs salaires sont nettement supérieurs à ceux des Sarajéviens. Sarajevo a dû s'adapter à ces nouveaux arrivants, et une double économie s'est mise en place : des boutiques, des logements ne sont aujourd'hui accessibles qu'aux plus hauts revenus, et sont souvent de fait réservés aux internationaux.

Enfin, la transition économique qui s'amorce actuellement en Bosnie-Herzégovine, et plus particulièrement à Sarajevo où la plupart des investissements se sont concentrés, n'est pas sans danger pour le pays et sa capitale. La question est de savoir si les entreprises bosniaques sauront faire face à cette nouvelle concurrence malgré leurs handicaps, ou si elles seront amenées à se marginaliser, voire disparaître, en laissant la place aux sociétés étrangères. Le risque est élevé, vu les grands groupes qui ont commencé à s'intéresser au marché potentiel que représente la Bosnie-Herzégovine. Volkswagen, BMW, Bouygues et Intermarché sont déjà sur cette liste. Aujourd'hui, symbole de la résistance de l'économie locale, un supermarché bosniaque, Cenex, s'est installé à Dobrinja, juste à côté de l'Interex ouvert par Intermarché. Mais combien de temps cette compétition pourra-t-elle être assumée ? Sarajevo n'est-elle pas en train de tomber, en même temps que le pays tout entier, sous le contrôle de puissances économiques extérieures?

\section{Conclusion}

Le destin de Sarajevo est encore incertain, tant au niveau national qu'international. Cette cité deviendra-t-elle une capitale de rang européen, à l'échelle modeste de ses 400000 habitants, ou ne sera-t-elle qu'un centre régional parmi d'autres, dans un pays «balkanisé » et dépendant du monde extérieur? L'homogénéisation ethnique actuelle n'est-elle qu'un épisode passager dans l'histoire mouvementée de Sarajevo, ou traduitelle une tendance plus durable? La diversité ethnique de Sarajevo a souvent été due, comme nous avons pu le constater, aux mesures politiques ou économiques de systèmes impériaux ou autoritaires. Aujourd'hui, une telle diversité ne peut se concevoir que dans le cadre d'un nouveau rayonnement économique de Sarajevo, dans 
un climat de tolérance réciproque entre les différentes communautés, et dans une Bosnie-Herzégovine solidaire et coopérante, sinon réunifiée. L'avenir de la capitale bosniaque se trouve ainsi lié à celui de la Bosnie-Herzégovine toute entière. En outre, il semble que seul un développement durable et contrôlé de la ville puisse permettre de résoudre les clivages ethniques ainsi que les différentes fractures sociales qui sont apparues ces dernières années au sein de la population sarajévienne.

\section{NOTES}

1. Voir Mudry (T.), Histoire de la Bosnie-Herzégovine. Faits et controverses, Paris : Ellipses, 1999, et Sanguin (A.-L.), «Sarajevo avant et après le siège, les mutations culturelles d'une capitale pluriethnique », Géographie et Cultures, (27), automne 1998, pp. 41-62.

2. Les retours minoritaires (minority returns) sont les retours de réfugiés ou de personnes déplacées dans des régions où leur communauté ethnique est minoritaire.

3. Sell (Louis), «The Serb Flight from Sarajevo : Dayton's First Failure », East European Politics and Societies, 14 (1), hiver 2000, pp. 179-202.

4. Federalni Zavod za Statistiku, Statistički podaci o privrednim / gospodarskim i drugim kretanjima u Federaciji Bosne i Herzegovina po kantonima, Sarajevo, mars 2000.

5. Ce sont respectivement les plans Vance-Owen (janvier 1993), Owen-Stoltenberg (juillet 1993), et le plan du "Groupe de contact" (juillet 1994).

INDEX

Index géographique : Bosnie-Herzégovine, Sarajevo

\section{AUTEUR}

\section{AMAËL CATTARUZZA}

Doctorant à l'Université de Paris-Sorbonne et chercheur associé au laboratoire CNRS-UPRESA 8064 « Espace et Culture » 